\title{
Clinical course of a critically ill patient with severe acute respiratory syndrome coronavirus 2 (SARS-CoV-2)
}

\author{
Nozomi Takahashi ${ }^{1} \cdot$ Ryuzo Abe $^{1} \cdot$ Noriyuki Hattori $^{1} \cdot$ Yosuke Matsumura $^{1} \cdot$ Taku Oshima $^{1} \cdot$ Toshibumi Taniguchi $^{2}$. \\ Hidetoshi Igari ${ }^{2} \cdot$ Taka-aki Nakada $^{1}$ (D)
}

Received: 4 April 2020 / Accepted: 9 June 2020 / Published online: 16 June 2020

(c) The Japanese Society for Artificial Organs 2020

\begin{abstract}
Although several studies have reported on the clinical and epidemiological characteristics of the patient with severe acute respiratory syndrome coronavirus 2 (SARS-CoV-2), clinical course of the most severe cases requiring treatment in ICU have been insufficiently reported. A 73-year-old man traveling on a cruise ship with history of hypertension and dyslipidemia developed high fever, dyspnea and cough after 7 days of steroid treatment for sudden sensorineural hearing loss, and tested positive for SARS-CoV-2 in sputa polymerase chain reaction (PCR) examination. His respiratory function deteriorated despite treatments with lopinavir/ritonavir, oseltamivir, azithromycin and meropenem at a regional hospital. He was intubated and transferred to the ICU in the tertiary university hospital on day 10 (ICU day 1). Interferon beta-1b subcutaneous injection was initiated immediately to enhance anti-viral therapy, and favipiravir on ICU day 10 upon availability. Progression of organ dysfunctions necessitated inhalation of nitrogen oxide for respiratory dysfunction, noradrenaline for cardiovascular dysfunction and continuous renal replacement therapy for renal dysfunction. His blood samples PCR also tested positive for SARS-CoV-2, indicating viremia, concomitantly with elevated IL-6 levels. VV-ECMO was initiated after sudden exacerbation of respiratory dysfunction on ICU day 7 to maintain oxygenation. The sustained excessive inflammatory cytokines in the present case might have led to the exacerbation of the disease, requiring vigorous organ support therapies to allow for survival and recovery from the rapid progression of multiple organ dysfunctions and severe respiratory failure.
\end{abstract}

Keywords SARS-CoV-2 $\cdot$ COVID-19 $\cdot$ VV-ECMO $\cdot$ Respiratory failure

$\begin{array}{ll}\text { Abbreviations } \\ \text { SARS-CoV-2 } & \begin{array}{l}\text { Severe acute respiratory syndrome corona- } \\ \text { virus } 2\end{array} \\ \text { COVID-19 } & \begin{array}{l}\text { Coronavirus disease } 2019 \\ \text { PCR }\end{array} \\ \text { Polymerase chain reaction } \\ \text { VV-ECMO } & \begin{array}{l}\text { Veno-venous extracorporeal membrane } \\ \text { oxygenation }\end{array}\end{array}$

Electronic supplementary material The online version of this article (https://doi.org/10.1007/s10047-020-01183-y) contains supplementary material, which is available to authorized users.

Taka-aki Nakada

taka.nakada@nifty.com

1 Department of Emergency and Critical Care Medicine, Chiba University Graduate School of Medicine, 1-8-1 Inohana, Chuo, Chiba 260-8677, Japan

2 Department of Infectious Diseases, Chiba University Hospital, Chiba, Japan

$\begin{array}{ll}\text { CRRT } & \text { Continuous renal replacement therapy } \\ \text { P/F ratio } & \mathrm{PaO}_{2} / \mathrm{FIO}_{2} \text { ratio } \\ \text { PEEP } & \text { Positive end-expiratory pressure } \\ \text { PPE } & \text { Personal protective equipment }\end{array}$

\section{Introduction}

Since the first report on an outbreak of a coronavirus disease 2019 (COVID-19) from China in December 2019, the disease has rapidly spread across the globe [1]; more than 693,000 cases were confirmed including more than 33,100 death (March 31, 2020) [2]. Although several studies have reported on the clinical and epidemiological characteristics of this disease, clinical course of the most severe cases requiring treatment in intensive care unit (ICU) have been insufficiently reported.

Here, we report a critically ill patient with viremia of severe acute respiratory syndrome coronavirus 2 
(SARS-CoV-2), who developed multiple organ dysfunctions, treated with artificial organ supports including mechanical ventilation, extracorporeal membrane oxygenation (ECMO) and continuous renal replacement therapy (CRRT).

\section{Case presentation}

A 73-year-old man was transferred to the ICU of our hospital for treatment of exacerbating respiratory failure due to COVID-19. His medical history included hypertension and dyslipidemia. While traveling on cruise ship, he experienced sudden sensorineural hearing loss and prednisone $40 \mathrm{mg}$ was initiated. Seven days later, he became febrile and developed dyspnea and cough. Due to the outbreak of COVID-19 on the cruise ship, polymerase chain reaction (PCR) test for SARS-CoV-2 using sputum sample was performed and turned out to be positive. Therefore, he was transferred from the cruise ship to a regional hospital according to the Japanese governmental quarantine policy. His respiratory status persisted without supplemental oxygen until day 4 after admission. Lopinavir/ritonavir was initiated on day 4 , since progressive ground glass opacity was detected in follow-up chest CT (Fig. 1a, Supplemental Figure EI). Supplemental oxygen was started on day 6 with $1 \mathrm{~L} / \mathrm{min}$. Oseltamivir, azithromycin and meropenem was added on day 8 in attempt to treat pneumonia causing deterioration of the respiratory function. His respiratory function slowly deteriorated requiring oxygenation with $15 \mathrm{~L} / \mathrm{min}$ on day 10 and body temperature persisted at $38^{\circ}$, while vital signs including consciousness and hemodynamics were stable. He was intubated and transferred to the ICU in the tertiary university hospital with ECMO specialists on day 10 (ICU day 1), due to deteriorating dyspnea and increasing demand for oxygen supplementation. He was transferred with a ventilator equipped with an anti-viral filter to reduce the risk of spreading infectious aerosol particles, and isolated in a negative pressure chamber in the ICU.

Upon ICU admission (day 10 from admission, ICU day 1 ), atelectasis in the dorsal side of the lung was noted in the chest CT (Fig. 1b, Supplemental Figure E2 and E3), while no apparent purulent sputum was observed by bronchoscopy, and presented no evidence of bacterial infection in the gram stain and culture. The $\mathrm{PaO}_{2} / \mathrm{FIO}_{2}(\mathrm{P} / \mathrm{F})$ ratio was 186 on $10 \mathrm{~cm} \mathrm{H}_{2} \mathrm{O}$ positive end-expiratory pressure (PEEP) without other organ dysfunctions (Fig. 2, Supplemental Table E1). Lopinavir/ritonavir was continued, and interferon beta- $1 \mathrm{~b}$ subcutaneous injection was initiated as anti-viral therapy considering the effect against RNA virus; oseltamivir, azithromycin and meropenem were discontinued. Various organ support therapies were initiated due to progressive organ dysfunctions, i.e. inhalation of nitric oxide for respiratory dysfunction (ICU day 2), noradrenaline for

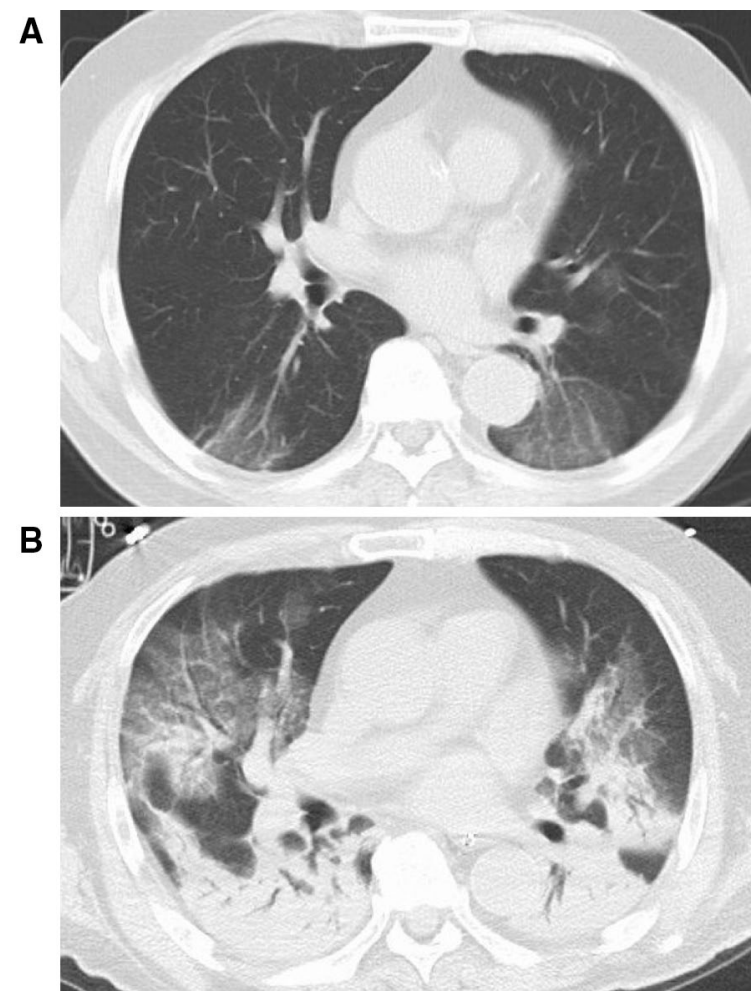

Fig. 1 Chest CT. a Chest CT (day 4). b Chest CT (day 10, ICU admission)

cardiovascular dysfunction (ICU day 3) and continuous renal replacement therapy for renal dysfunction (ICU day 4). PCR tests for SARS-CoV-2 using blood samples were positive (ICU day 5 and 6), indicating viremia. Sustained high blood IL-6 level and sudden exacerbation of respiratory dysfunction on ICU day 7 (P/F ratio 72 on $24 \mathrm{~cm} \mathrm{H}_{2} \mathrm{O}$, airway pressure release ventilation) (Supplemental Figure E4) made initiation of VV-ECMO inevitable to maintain his oxygenation (blood flow, $4 \mathrm{~L} / \mathrm{min}$ at $2700 \mathrm{rpm}$ by a centrifugal pump [Rotaflow ${ }^{\circledR}$, Maquet Getinge, Rastatt, Germany]; sweep gas flow, $4 \mathrm{~L} / \mathrm{min}$; artificial lung [BIOCUBE 6000®, Nipro, Osaka, Japan]; drainage cannula [25Fr. HLS cannula ${ }^{\circledR}$, Maquet Getinge, Rastatt, Germany], placed in right atrium through right jugular vein; outflow cannula [19Fr. HLS cannula ${ }^{\circledR}$, Maquet Getinge, Rastatt, Germany] in right femoral vein). Progressive organ dysfunctions presented the worst condition on ICU day 11. Although daily sputum examination proved bacterial infection unlikely, antibacterial agent was switched from cefepime to meropenem to broaden treatment spectrum. On ICU day 10, Chest X-ray presented white-out appearance, indicating complete atelectasis with no detectable tidal volume by ventilator (Supplemental Figure E5), and favipiravir supplied by Japanese government was initiated to replace Lopinavir/ritonavir. The CRRT support allowed adjusting the rigorous water balance and VVECMO allowed lung rest which avoid pulmonary damage. 
Fig. 2 Clinical course

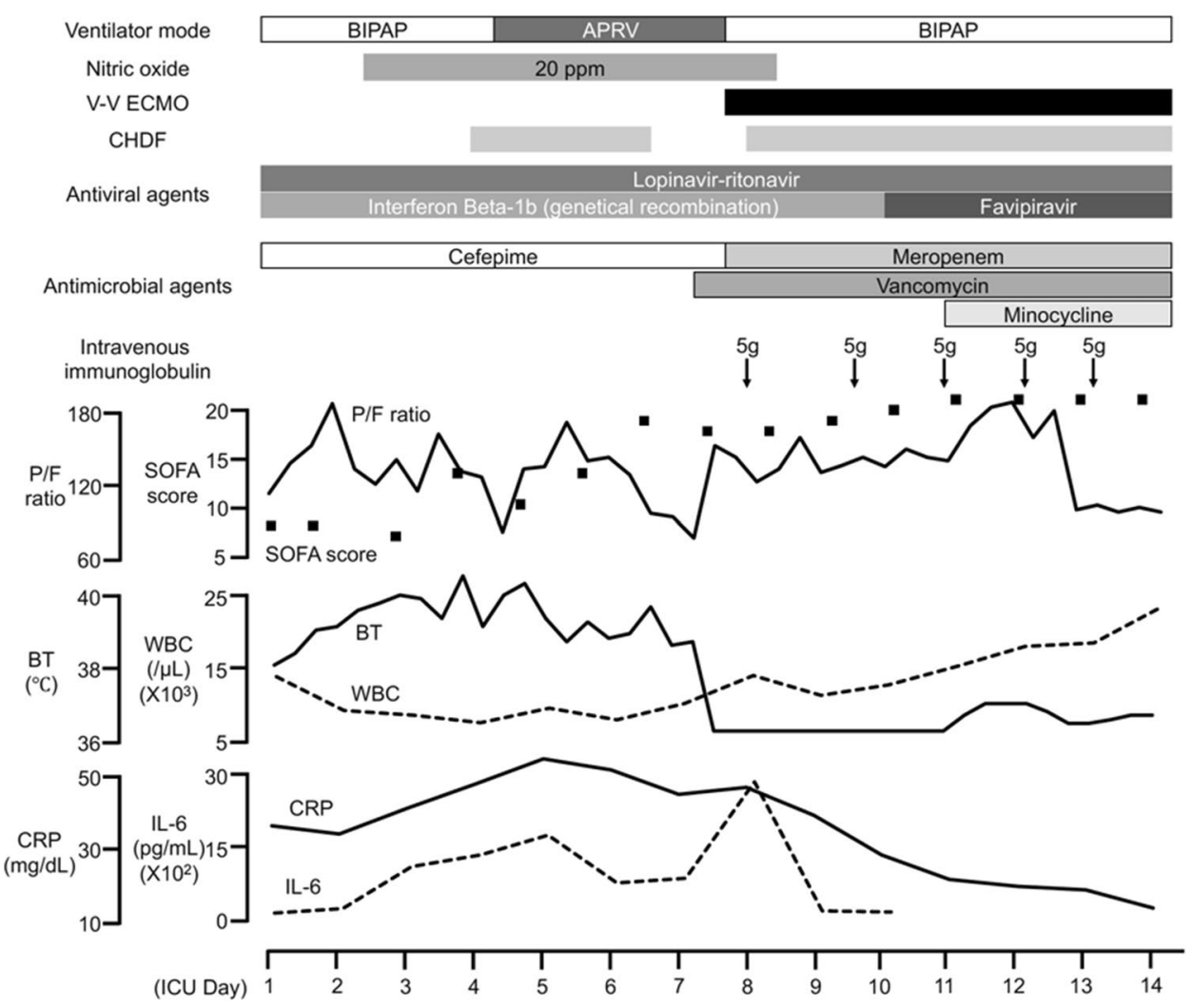

On ICU day 13, gradual recovery from complete atelectasis was noted in chest X-ray (Supplemental Figure E6). Despite vigorous supportive care including prone positioning, chest CT on ICU day 53 showed diffuse alveolar damage depicting irreversible change of the lung (Supplemental Figure E7). Further treatments were withheld and the patient deceased on ICU day 59.

\section{Discussion}

Here we reported the severe case with viremia of SARSCoV-2, who admitted to the ICU on Day 10. He presented progressive multiple organ dysfunctions with sustained blood IL-6 level and required various artificial organ support therapies (vasopressor [ICU day 3], CRRT [ICU day 4], VV-ECMO [ICU day 7]). On ICU day 13, gradual recovery initiated.

Excessive humoral mediator activity including inflammatory cytokines were suggested to be a key component of the pathophysiology in severe cases of COVID-19 [3-5]. The present case also presented elevated blood IL-6 levels, which sustained at 142-2667 pg/mL during initial 8 ICU days. High fever persisted with elevated blood IL-6 levels, until temperature management with ECMO. Such exaggerated inflammatory reaction might be induced by high viral load, suggested by sustained viremia confirmed by positive blood PCR tests for SARS-CoV-2 on ICU day 5 and 6, concomitantly with high IL-6 levels. Involvement of viremia has also been reported in a case-series from Wuhan of 41 patients including 13 in the ICU with laboratory-confirmed 2019-nCoV infection, 6 patients (15\%) had positive PCR tests for SARS-CoV-2 using blood samples [3]. However, the highest level of IL- 6 on ICU day 8 might reflect the complication of bacterial infection.

Favipiravir, an antiviral drug approved as a stockpile against influenza pandemics in Japan, was distributed as an option for anti-viral therapy against SARS-CoV-2 under government control. The efficacies of antiviral therapies have not been clarified in the clinical course of the patients. However, since the recovery became evident 2 days after favipiravir treatment in the present case, favipiravir may have contributed to the amelioration of the lung lesion. The case was registered in a clinical study of favipiravir, which would provide the efficacy.

Beneficial effects of ECMO for respiratory failure were reported during the influenza $\mathrm{A}(\mathrm{H} 1 \mathrm{~N} 1)$ pandemic in 2009 [6], especially in expert centers with ECMO specialists $[7,8]$. In Japan, a committee of ECMO project was established after the pandemic to promote education of ECMO management and construction of referral network, and has led to an increase in the number of ECMO specialists and better outcomes [9]. In the present case, the transfer of critical case to the regional ICU with ECMO specialists 
was well managed by the ECMO network physicians. The implementation of ECMO was performed in the negative pressure isolation room with standard sterile precautions in addition to the full personal protective equipment (PPE) including goggles, N-95 face mask, hood and gown. Poor vision through the goggles and hood, and hot and humid environment induced by full PPE increased the difficulty of ultrasound-guided insertion of the cannula. Briefing of the procedures were essential to achieve the safe and smooth ECMO implementation.

\section{Conclusions}

In the present case, excessive inflammatory cytokines sustained over a week after ICU admission, possibly leading to the exacerbation of the disease. Vigorous organ support therapies were mandatory to allow for survival and recovery from the rapid progression of multiple organ dysfunctions and severe respiratory failure.

Author contributions NT, RA, NH, YM, TO, TT, HI and TN contributed to study conception, data acquisition, data interpretation, manuscript drafting, and critical revision of the manuscript for important intellectual content. All authors read and approved the final manuscript.

Funding None.

\section{Compliance with ethical standards}

Conflict of interest The authors have no conflicts of interest to declare
J Med. 2020. https://doi.org/10.1056/NEJMoa2002032(Epub ahead of print).

2. WHO. Coronavirus disease (COVID-2019) situation reports. March 17, 2020. https://www.who.int/emergencies/diseases/novel -coronavirus-2019/situation-reports/. Accessed 31 Mar 2020.

3. Huang C, Wang Y, Li X, Ren L, Zhao J, Hu Y, et al. Clinical features of patients infected with 2019 novel coronavirus in Wuhan China. Lancet. 2020;15:497-506.

4. Puja M, Daniel F, Michael B, Emilie S, Rachel ST, Jessica J. COVID-19: consider cytokine storm syndromes and immunosuppression. Lancet. 2020;395:1033-4.

5. Qiurong R, Kun Y, Wenxia W, Lingyu J, Jianxin S. Clinical predictors of mortality due to COVID-19 based on an analysis of data of 150 patients from Wuhan China. Intensive Care Med. 2020;3:1-3.

6. Davies A, Jones D, Bailey M, Beca J, Bellomo R, Blackwell $\mathrm{N}$, et al. Extracorporeal membrane oxygenation for 2009 influenza A (H1N1) acute respiratory distress syndrome. JAMA. 2009;302:1888-955.

7. Barbaro RP, Odetola FO, Kidwell KM, Paden ML, Bartlett RH, Davis MM, et al. Association of hospital-level volume of extracorporeal membrane oxygenation cases and mortality. Analysis of the extracorporeal life support organization registry. Am J Respir Crit Care Med. 2015;191:894-901.

8. Noah MA, Peek GJ, Finney SJ, Griffiths MJ, Harrison DA, Grieve $\mathrm{R}$, et al. Referral to an extracorporeal membrane oxygenation center and mortality among patients with severe 2009 influenza A (H1N1). JAMA. 2011;306:1659-68.

9. Ohshimo S, Shime N, Nakagawa S, Nishida O, Takeda S. Comparison of extracorporeal membrane oxygenation outcome for influenza-associated acute respiratory failure in Japan between 2009 and 2016. J Intensive Care. 2018;6:38.

Publisher's Note Springer Nature remains neutral with regard to jurisdictional claims in published maps and institutional affiliations.

\section{References}

1. Guan WJ, Ni ZY, Hu Y, Liang WH, Ou CQ, He JX, et al. Clinical Characteristics of Coronavirus Disease 2019 in China. N Engl 